\title{
NASOADHESIVE DRUG DELIVERY: A REVIEW
}

\author{
${ }^{1}$ Gupta AK $*,{ }^{2}$ Siddiqui AW, ${ }^{3}$ Tiwari PK, ${ }^{3}$ Rajpoot SS, ${ }^{3}$ Yadav C, ${ }^{3}$ Singh K \\ ${ }^{1}$ Micro Labs ltd, Baddi, Himachal Pradesh, INDIA \\ ${ }^{2}$ Department of Pharmacy, Lloyd Institute of Management \& Technology, Greater Noida, INDIA \\ ${ }^{3}$ Department of Pharmacy, Harlal Institute of Management \& Technology, Greater Noida, INDIA \\ *Corresponding Author's E-mail:anishpharma02@yahoo.co.in, Mob. +91-9312621718 \\ Received 23 Dec 2011; Revised 20 Jan 2012; Accepted 20 Jan 2012, Available online 20 Jan 2012
}

\begin{abstract}
Nasal route has been demonstrated as being a possible alternative to the intravenous route for the systemic delivery of drugs. It has been demonstrated that low absorption of drugs can be enhanced by using absorption enhancers or increasing the drug residence time in the nasal cavity, by using mucoadhesive polymers, which serve both functions. The factors that influence mucoadhesiveness of a polymer include physiological factors polymer related factors and environmental factors are briefly discussed in present review. Applications of nasoadhesive drug delivery of small organic molecules, antibiotics, proteins, and vaccines are discussed. Furthermore, nasal dosage forms and their effect on drug delivery also discussed.
\end{abstract}

Keywords: Nasal drug delivery, Mucin, Mucoadhesive polymers, Bioadhesion

\section{INTRODUCTION}

Nasal route has been demonstrated as being a possible alternative to the intravenous route for the systemic delivery of drugs. In addition to rapid absorption and avoidance of hepatic first-pass metabolism, the nasal route allows the preferential delivery of drug to the brain via the olfactory region, and is therefore a promising approach for the rapid-onset delivery of medications. ${ }^{1}$

Therapy through intranasal administration has been an accepted form of treatment in the Ayurvedic system of Indian Medicine. In recent years many drugs have been shown to achieve better systemic bioavailability through nasal route than by oral administration. ${ }^{2}$

In modern pharmaceutics, the nose had been considered primarily as a route for local drug delivery. Advances in biotechnology have made available a large number of protein and peptide drugs for the treatment of a variety of diseases. These drugs are unsuitable for oral administration because they are significantly degraded in the gastrointestinal tract or considerably metabolized by first pass effect in the liver. Even the parenteral route is inconvenient for long term therapy. Of the many alternate routes tried, intranasal drug delivery is found much promising for administration of these drugs. ${ }^{3}$

The drugs showing poor bioavailabilities, typically in the order of $5-10 \%$ for large molecules. On the other hand, very good results were obtained with small organic molecules. ${ }^{4}$ The causes of failure led to the conclusion that the short residence time of the formulation within the nasal cavity coupled to the low permeability. Consequently, the attention shifted to the evaluation of mucoadhesive polymers, some of which would even demonstrate additional permeation- enhancing capabilities. ${ }^{5,6}$ The encouraging results and the desire to overcome some new challenges stimulated the development of new generations of polymers based on $\mathrm{pH}$ or thermal responsiveness or modified existing polymers having improved bioadhesive or permeation-enhancing properties. ${ }^{7,8,9}$ Even though a number of challenges are still to be overcome, especially with respect to toxicity, the potential of nasal drug delivery (NDD), including the ability to target drugs cross the blood-brain barrier (BBB), are very high and continues to stimulate academic and industrial research groups so that we will keep witnessing increasing number of advanced nasal drug delivery products.

\section{NASAL ANATOMY AND PHYSIOLOGY}

The nasal cavity is divided into two halves by the nasal septum and extends posteriorly to the nasopharynx, while the most anterior part of the nasal cavity, the nasal vestibule, opens to the face through the nostril (Fig. 1). The atrium is an intermediate region between the vestibule and the respiratory region. The respiratory region, the nasal conchae or turbinates, which occupies the major part of the nasal cavity, possesses lateral walls dividing it into 3 sections: the superior, middle and inferior nasal turbinates.

The nasal vestibule has the smallest cross-sectional area in the respiratory tract (approximately $0.3 \mathrm{~cm}^{2}$ on each side) that extends from the entrance of nostril, which is guarded by vibrissae (hairs), to the anterior end of the inferior turbinate. The area from the anterior ends of the turbinate to the anterior portion of the nasopharynx constitutes the main nasal passages. ${ }^{10,11}$ The epithelial cells in the nasal vestibule are stratified, squamous and keratinized with sebaceous glands (Fig.2). Due to its nature, the nasal vestibule is very resistant to dehydration and can withstand noxious environmental substances and limits permeation of substances. Microvilli are found on the columnar cell, 
which increases the surface area available for absorption. The nasal mucosa is highly vasculature, superficial and deep layers of arterioles supply the lamina propria and between the venules and capillaries. Most of the area of the nasal cavity serves the function of cleaning the air we breathe before it reaches the lungs. It does this with the help of the respiratory mucosa, which lines the walls of the nasal cavity. Within this mucosa, small, hair-like cilia move in a wave-like motion, moving mucus to the back of the throat. ${ }^{10}$
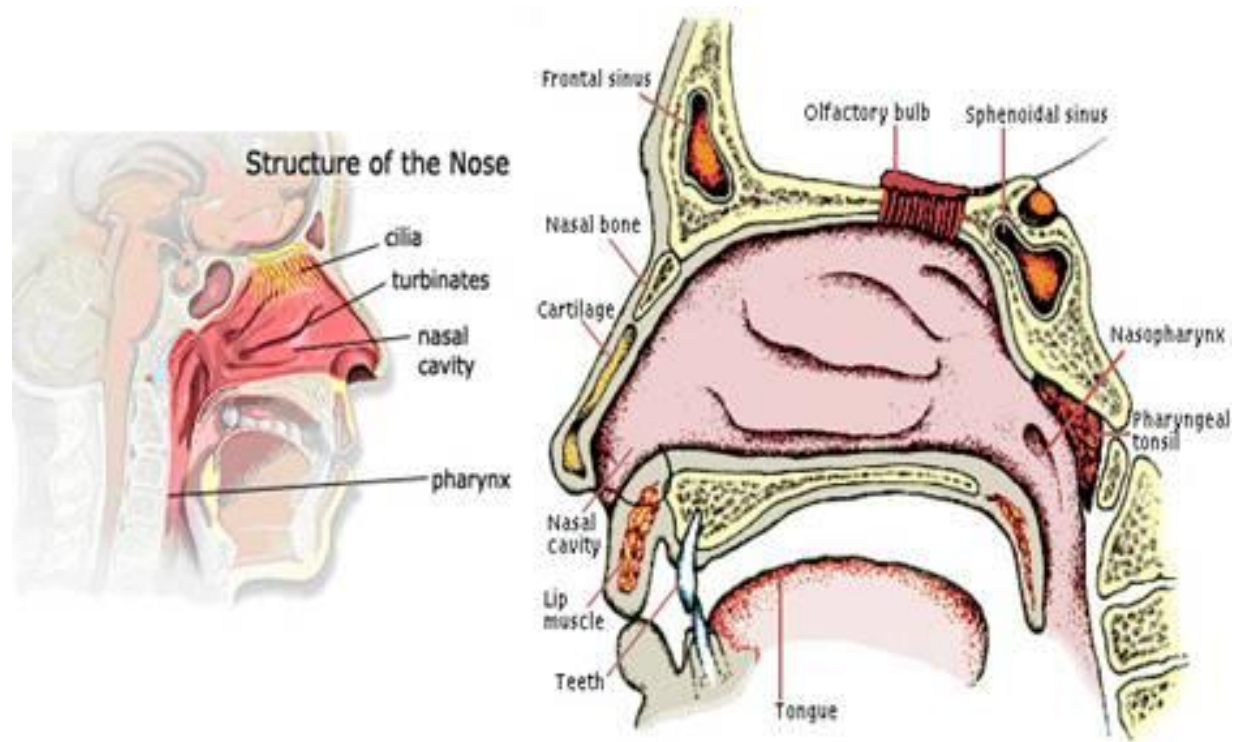

Figure 1: Structure of nasal cavity

\subsection{Nasal mucus secretion:}

The submucosal glands, which secrete the greater quantity of nasal mucus, comprise both mucus cells, secreting the mucus gels, and serous cells, producing a watery fluid. ${ }^{12}$ Seromucus glands in the human nose have been estimated to be $100,000 .{ }^{13}$ Mucus is also released from the goblet cells as mucus granules, which swell in the nasal fluids to contribute to the mucus layer. Mucus secretion is a complex mixture of many substances and consists of about $95 \%$ water, $2 \%$ mucin, $1 \%$ salts, $1 \%$ of other proteins such as albumin, immunoglobulins, lysozyme and lactoferrin, and $<1 \%$ lipids. ${ }^{14}$ About $1.5-2$ L of nasal mucus is produced daily. This mucus blanket, about $5 \mathrm{~mm}$ thick, consists of two layers, a lower sol layer and an upper gel layer. The viscosity of both layers affects ciliary beating and the efficiency of transporting the overlying mucusthe mucociliary clearance (MCC). The nasal mucus performs a number of physiological functions. (1) It covers the mucosa, and physically and enzymatically protects it. (2) The mucus has water-holding capacity. (3) It exhibits surface electrical activity. (4) It permits efficient heat transfer. (5) It acts as adhesive and transports particulate matter towards the nasopharynx.

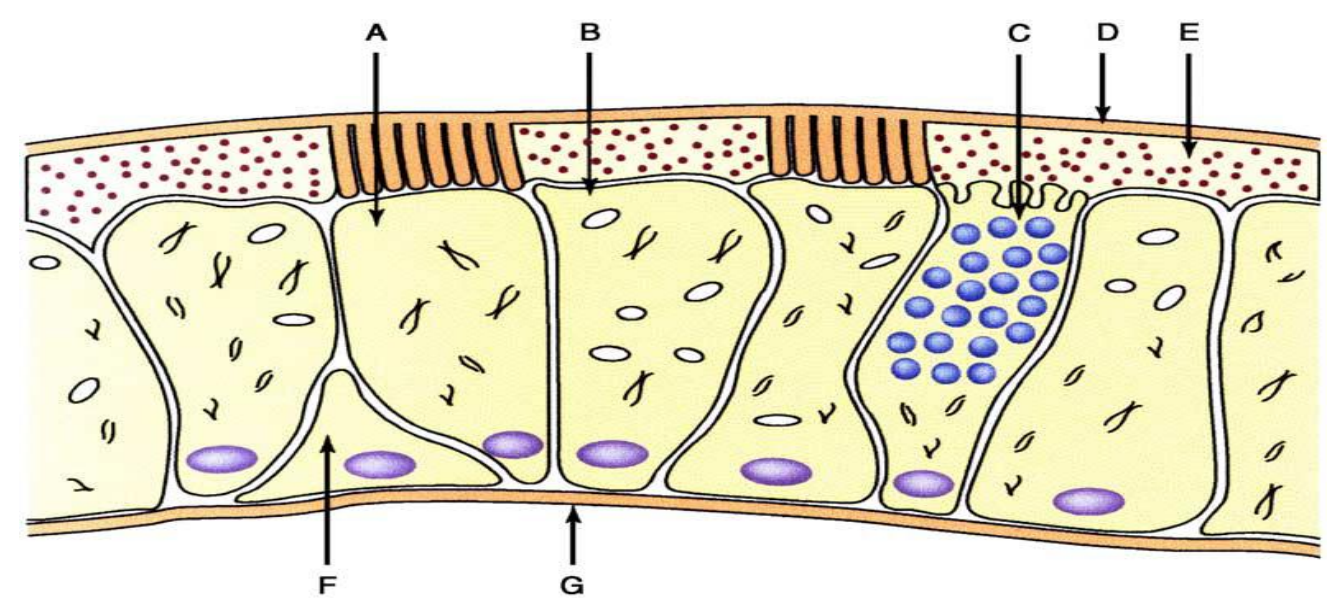

Figure 2: Nasal epithelium showing ciliated cell (A), non-ciliated cell (B), goblet cells (C), gel mucus layer (D), sol layer $(\mathrm{E})$, basal cell $(\mathrm{F})$ and basement membrane $(\mathrm{G})$. 


\subsection{Mucociliary clearance:}

One of the functions of the upper respiratory tract is to prevent noxious substances (allergens, bacteria, viruses, toxins etc.) from reaching the lungs. When such materials adhere to, or dissolve in the mucus lining of the nasal cavity, they are transported towards the nasopharynx for eventual discharge into the GIT. Clearance of this mucus and the adsorbed/dissolved substances into the GIT is called the MCC. It consists of a coordinated interaction between the overlying mucus layer and the methachronal wavelike movement of the underlying cilia. Cilia are hairlike protrusions on the epithelial cell surface. There are approximately 300 cilia per cell, each cilia is $5-10 \mathrm{~mm}$ long and $0.1-0.3 \mathrm{~mm}$ wide and beats at a frequency of about $20 \mathrm{~Hz}$.

The MCC can be influenced by environmental and pathological conditions. Factors that can increase ciliary beat frequency $(\mathrm{CBF})$ and mucus production, or decrease mucus viscosity will all lead to increase in MCC. Environmental conditions like temperature (below or above $23^{\circ} \mathrm{C}$ ), inhalation of sulfur dioxide and cigarette smoke all decrease MCC. ${ }^{14,15}$ Such pathological conditions like Sjorgens syndrome, asthma, nasal polyposis, defective septum, rhinitis, allergy, common cold and sinusitis alter MCC due to their effects on CBF or mucus rheology. Two mechanisms have been suggested to be involved in increased mucus secretion irrespective of the causative factors. First, mast cell-derived mediators released in the nasal mucosa may induce secretion of nasal mucus glycoproteins. Second, neurohormones released may cause an increase in nasal blood flow and increase in transudation of fluid and plasma proteins. ${ }^{16}$ All the pathological and environmental conditions above will ultimately alter nasal drug delivery and the performance of nasal mucoadhesive formulations, and should be taken into account during product development.

\subsection{Nasal mucus \& mucus-drug interaction:}

Nasal mucus is produced by the nasal mucosa, and mucal tissues lining the airways (trachea, bronchus, bronchioles) is produced by specialized airway epithelial cells (goblet cells) and submucosal glands. Small particles such as dust, particulate pollutants, and allergens as well as infectious agents such as bacteria are caught in the viscous nasal or airway mucus and prevented from entering the system. This event along with the continual movement of the respiratory mucus layer toward the oropharynx, helps prevent foreign objects from entering the lungs during breathing. Additionally, mucus aids in moisturizing the inhaled air and prevents tissues such as the nasal and airway epithelia from drying out. ${ }^{17}$ Nasal and airway mucus is produced constitutively, with most of it swallowed unconsciously, even when it is dried. ${ }^{18}$

Table 1: Different sections of nasal cavity and their relative permeability

\begin{tabular}{|cll|}
\hline S. No. & Region & Permeability \\
\hline $\mathbf{1 .}$ & Nasal Vestibule & Least permeable because of presence of keratinized cells \\
$\mathbf{2 .}$ & Atrium & Less permeable as it has small surface area and stratified cells are present \\
3. & Respiratory Region & Most permeable region because of large surface area and rich vasculature \\
$\mathbf{4 .}$ & Olfactory Region & Direct access to cerebrospinal fluid \\
$\mathbf{5 .}$ & Nasopharynx & Receives nasal cavity drainage \\
\hline
\end{tabular}

Increased mucus production in the respiratory tract is a symptom of many common illnesses, such as the common cold and influenza. Similarly, hypersecretion of mucus can occur in inflammatory respiratory diseases such as respiratory allergies, asthma, and chronic bronchitis. ${ }^{17}$ The presence of mucus in the nose and throat is normal, but increased quantities can impede comfortable breathing and must be cleared by blowing the nose or expectorating phlegm from the throat. Tears are also a component of nasal mucus.

Mucin is the major component of mucus. This compound, primarily responsible for the viscoelastic properties of the mucus, is found in 2 forms: soluble secretory mucin and membrane-bound mucin. ${ }^{16,19}$ Mucins are heterogenous macromolecules composed of approximately $10-30 \%$ by weight of peptide core linked to oligosaccharide chains that make up $70-80 \%$ of the total weight. ${ }^{20}$

It is an important consideration to understand how the mucus and drugs interact with each other, and even more importantly how such interactions could influence drug absorption and activity. The drug's molecular size, the mucus mesh size and the interaction between the mucus gel and the drug influence the diffusion of drugs through the mucus. Like in all cases of diffusion, small and neutral molecules diffuse faster and to a greater extent through a charged matrix compared to charged and large molecules. It should be highlighted that some large or charged molecules have been observed to diffuse readily through the mucus. $^{21}$ Additionally, particulate materials, e.g., microspheres and liposomes bearing drugs, have been observed to diffuse through the mucus and reach the epithelium intact.

Studies on particulate transport through the mucus all demonstrated that diffusion is size dependent and decreases quickly with increasing size. ${ }^{22}$

\subsection{Polymer-mucus interaction:}

Several theories have been put forward to explain the mechanism of polymer-mucus interactions that lead to mucoadhesion. The sequential events that occur during bioadhesion include an intimate contact between the bioadhesive polymer and the biological tissue due to proper wetting of the bioadhesive surface and swelling of the bioadhesive polymer, this leads to the penetration of the bioadhesive into the tissue crevices, interpenetration between the mucoadhesive polymer chains and those of the mucus. Subsequently low chemical bonds can become operative. $^{23,24}$ Hydration of the polymer plays a very important role in bioadhesion. There is a critical degree of hydration required for optimum bioadhesion. If there is incomplete hydration, the active adhesion sites are not 
completely liberated and available for interaction. On the other hand, an excessive amount of water weakens the adhesive bond as a result of an overextension of the hydrogen bonds. During hydration, there is a dissociation of hydrogen bonds of the polymer chains. The polymerwater interaction becomes greater than the polymerpolymer interaction, thereby making the polymer chains available for mucus penetration. ${ }^{25}$

The adsorption theory of bioadhesion proposes that adhesion of a polymer to a biological tissue results from: (1) primary chemical bonds that are somewhat permanent and therefore undesirable in bioadhesion, (2) Vander Waals, hydrogen, hydrophobic and electrostatic forces, which form secondary chemical bonds. ${ }^{26}$ The electronic theory proposes the existence of an electrical charge double layer at the interface between the adhesive and biological tissue as a result of the difference in their electronic structure. ${ }^{27}$

\subsection{Factors that influence mucoadhesion:}

The factors that influence mucoadhesiveness of a polymer includes, physiological factors polymer related factors and environmental factors.

\subsubsection{Physiological-related factors:}

MCC, mucus turnover and disease states are physiological factors which influence nasal mucoadhesion. Mucoadhesion can slow down MCC, but with time mucus production reduces the mucoadhesion bond strength, allowing a recovery of MCC to normal clearance rates, thereby removing the mucoadhesive. Disease conditions mentioned earlier can affect mucoadhesion due to their influence on either mucus production or ciliary beating. Thus a good understanding of the nature of mucus in these diseases is imperative in designing a good novel drug delivery (NDD) system. An abnormal mucus layer could present an unanticipated barrier to drug transport through the mucosa. Mucoadhesive capabilities of polymers should be studied during product development under such disease conditions considered relevant.

\subsubsection{Polymer-related factors:}

The polymer molecular mass will influence its bioadhesion characteristics. There is a critical polymer molecular mass and cross-linking density below or above which there is reduced adhesive power, and this varies with the type of polymer. $^{28,29}$ Mucoadhesion requires an adequate free chain length for interpenetration to occur. Reducing the free chain length by extensive cross-linking therefore reduces mucoadhesion. ${ }^{28,30}$ An optimum polymer concentration is required at the polymer-mucus interface for bioadhesion, beyond which few polymer chains will be available for polymer-mucus interpenetration. The polymer concentration that is required for optimum bioadhesion is different between gels and solid bioadhesives. In the liquid state, an optimum concentration exists for each polymer beyond that reduced adhesion results because fewer polymer chains will be available for interpenetration with the mucus. On the other hand, with solid dosage forms such as buccal tablets, increased polymer concentration leads to increased mucoadhesive power. ${ }^{24,31,32}$

\subsubsection{Environmental factors:}

Polymer hydration and swelling are required for initiation of mucoadhesion but excessive hydration with inordinate swelling of the polymer reduces its adhesive strength. The swelling/hydration rate should not be too rapid in order to prolong the adhesion time.

On the other hand, inordinate swelling is eventually required to reduce polymer adhesiveness and to allow it to detach from the biological tissue. Some polymers owe their mucoadhesiveness to such forces as hydrogen bonding, van der Waals, hydrophobic and electrostatic forces. The strength of these forces is influenced by the environmental $\mathrm{pH}$.

Consequently, for such polymers, environmental $\mathrm{pH}$ is a very important determinant of mucoadhesive strength.

\section{NASAL DOSAGE FORM AND DELIVERY SYSTEM}

The final dosage form used for nasal drug delivery is chosen after consideration of a wide range of factors, covering patient convenience, efficiency of drug delivery and formulation reasons. Nasal sprays, squeeze bottles, and liquid droppers are some of the more common delivery methods that can be seen as nasal dosage forms. There are three main ways of depositing inhaled particles or the nasal lining: impaction, sedimentation and diffusion. Impaction occurs when there is a change in direction of the airflow as happens when inspired air passes through the nasal valve - and the inertia of large or fast-moving particles carries them in their original direction. Sedimentation happens when the moving slowly and the particles settle slowly under the force of gravity. The final method of deposition diffusion occurs by Brownian motion and is thus limited to very small particles $(<0.5 \mathrm{~mm})$. Nasal dosage forms will usually contain the drug in a liquid or powder formulations delivered by a pressurized or pump system.

Table 2: Summary of some nasal drug delivery studies where mucoadhesive polymers were employed

\begin{tabular}{|cllll|}
\hline S.No. & Drug & Mucoadhesive polymer & Dosage form & Animal species \\
\hline 1. & Gentamicin & Chitosan & Powder & Rabbit \\
2. & Ciprofloxacin & HPMC & Gel & Rabbit \\
3. & Insulin & Chitosan glutamate & Liquid & Sheep \\
4. & Insulin & Maltodextrin DE 22 & Powder & Rabbits \\
$\mathbf{5 .}$ & Apomorphine & Carbopol 971P & Powder & Rabbits \\
6. & Pentazocine & Chitosan microspheres & Powders & Rabbits \\
7. & Dopamine & Hydroxypropyl cellulose & Solution & Dogs \\
8. & Metoprolol tartrate & Alginate microspheres & Liquid & Rabbits \\
\hline
\end{tabular}


Liquid formulations used for nasal drug delivery are usually aqueous solutions of the drug and thus have the general benefits and drawbacks of pharmaceutical solutions. They are relatively simple to develop and manufacture compared to solid dosage forms but often have a lower microbiological and chemical stability, requiring the use of various preservatives. Squeezed bottles are often used for nasal decongestants and work by spraying a partially atomized jet of liquid into the nasal cavity. They give a better absorption of drug by directing the formulation into the anterior part of the cavity and covering a large part of the nasal mucosa. Metered-dose pump systems offer greater control over dosing than any of the previous systems discussed above. They can deliver solutions, suspensions or emulsions, with a predetermined volume between 25 and $200 \mathrm{~mL}$, thus offering deposition over a large area. Particle size and dose volume are two, important factors for controlling delivery from metereddose systems. Nasal sprays are available as squeeze bottles, which would not be expected to give reproducible dosing. They are also available as metered-dose devices, which would be expected to give more reproducible dosing, as a mechanical actuation delivers a predetermined volume to the patient. Nasal drops rely upon the instillation of one or more drops of drug solution, either from a dropper with a flexible (rubber) teat, or directly from a "squeezable" plastic container into the nasal cavity.

Many conventional drugs have been investigated for systematic delivery via the nasal cavity and these are of relatively low molecular weight (in most cases less than $500 \mathrm{Da}$ ). However, peptides and proteins generally have a molecular weight in excess of 1,000 Da and are therefore unlikely to be absorbed across the nasal mucosa in any appreciable amounts without pharmaceutical intervention. New technologies in nasal delivery are primarily concerned with strategies to increase drug absorption. Drug absorption can be increased by one of the techniques like use of surfactant increasing the permeability of nasal epithelium, altering the mucus layer, altering the tight junctions, reverse micelle formation, erosion of mucosal layer, increasing contact time at the site of absorption, modifying the site of deposition, use of bioadhesives, reducing rate of mucociliary clearance and inhibition of enzymatic degradation. ${ }^{33}$

Apart from liquid, powder and gels some novel drug delivery system are being used to deliver drug via nasal route. This drug delivery system includes microspheres ${ }^{34}$, liposomes and nanoparticles. ${ }^{35}$ Microsphere technology is one of the specialized systems becoming popular for designing nasal products, as it may provide prolonged contact with the nasal mucosa and thus enhances absorption and bioavailability. In the presence of microspheres, the nasal mucosa is dehydrated due to moisture uptake by the microspheres. This result in reversible shrinkage of the cells, providing a temporary physical separation of the tight (intercellular) junctions that increases the absorption of the drugs. ${ }^{36}$ Particularly important for the nasal drug absorption is the respiratory region, which contains three nasal turbinates and the deposition of the particles in this region will depend on their size. Classically, larger particles including droplets
$(>10 \mu \mathrm{m})$, are deposited in the nasal cavity after inhalation, the larger the particles, the more anterior the deposition. For smaller particles the site of deposition depends on the velocity at which the particles are inhaled and the turbulence in the air flow, however the particles of size smaller than $1 \mu \mathrm{m}$ are not normally deposited in the nasal cavity but travel down to the trachea to reach the lung. The rationale behind the use of a microsphere system is that, the application of bioadhesive microspheres (in the powder form) with good bioadhesive properties would permit such microspheres to swell in contact with nasal mucosa to form a gel and control the rate of clearance from the nasal cavity, thereby giving poorly absorbed drugs a longer time to be available at the absorptive surface. Microspheres have been reported to be present up to $3-5 \mathrm{~h}$ in the nasal cavity depending upon the bioadhesive material used for formulation. The ideal microsphere particle size requirement for nasal delivery should range from 10 to 50 $\mu \mathrm{m}$ as smaller particles than this will enter the lungs.

Nanoparticles are solid colloidal drug delivery system, submicron in size from 1-1000 $\mu \mathrm{m}$. The drug molecules are either encapsulated or adsorbed on the nanoparticles. They possess better reproducibility and stability profile than liposomes. They offer non-invasive route of administration via nasal route also. Nanoparticles have been used to deliver drugs as well as vaccines through nasal route. ${ }^{35}$

\section{APPLICATION OF NASOADHESIVE DRUG DELIVERY IN PHARMACEUTICALS}

\subsection{Antibiotics:}

Recently a few studies have examined the potential of the nasal route for systemic delivery of antibiotics using mucoadhesive polymers. In a preliminary study, Lim et al. 37 prepared and evaluated mucoadhesive microspheres of hyaluronic acid and chitosan for nasal delivery of gentamicin and other drugs. The study showed that hyaluronic acid and hyaluronic acid/chitosan microspheres could adhere to the nasal mucus. Subsequently, the authors showed that hyaluronic acid and chitosan may be employed for nasal administration of antibiotics to obtain a high bioavailability and prolonged release. ${ }^{38}$ Successful nasal delivery of other antibiotics such as vancomycin and tobramycin with chitosan has been reported as well. ${ }^{39} \mathrm{It}$ was shown that the presence of chitosan salts slow down the release of vancomycin hydrochloride at $\mathrm{pH} 5.5$ and $\mathrm{pH}$ 7.4.

Although it is very interesting to deliver antibiotics through the nasal route, the use of mucoadhesives raises important safety concerns due to the prolonged drugpolymer residence time in the nasal mucosa, which may lead to superinfection in the respiratory tract. The increased contact time may upset the nasal normal microbial flora when broadspectrum antibiotics are used. This may lead to superinfection as documented for the gastrointestinal mucosa. This should be considered when selecting antibiotics to be delivered nasally using mucoadhesives.

\subsection{Macromolecules:}

\subsubsection{Vaccines and DNA:}


Vaccination through nasal cavity is another interesting tool for pathogenic infections such as influenza, pertussis, meningitis, measles, etc.. The route of administration is easy, cheap and can be administered to a large population. It eliminates the use of needles and as such risk of infections (especially in poor countries) with hepatitis B, $\mathrm{HIV}$, etc. There is a relatively large surface area through which uptake of the antigenic material can take place. Mucosal immunization may be safer and more successful in young children in the presence

of marternal antibodies as demonstrated by the high effectiveness of the live polio vaccine when given at birth. A recent review article by Lemoine et al. ${ }^{40}$ summarized vaccination using nasally administered microparticles. In the studies cited, various levels of immune responses were induced. Vila et al. $^{41}$ studied nasal immunization with tetanus toxoid following encapsulation and administration in the forms of PEG-coated polylactic acid nanospheres, chitosan-coated polylactic- glycolic acid nanospheres and chitosan nanospheres. They observed that PEG-coated nanospheres induced higher levels of tetanus toxoid in the blood compared to chitosan-coated nanospheres. On the other hand, very high $\operatorname{IgG}$ titers were obtained 6 months post administration of chitosan nanospheres.

\subsubsection{Proteins:}

Low absorption of the compounds such as high molecular weight proteins occurs due to, rapid mucociliary clearance and enzymatic degradation. Some mucoadhesive polymers have been suggested to extend residence time and improve uptake of large molecules across the nasal mucosa. ${ }^{42}$ Nonprotein high molecular weight compounds (e.g., mannitol, cyanocobalamin) have been used as surrogates in search of mucoadhesives for nasal administration of proteins. ${ }^{43}$ Such

\section{REFERENCES}

1. Vladimir PT. Nanoparticulate As Drug Carriers. Imperial College Press,London, 2006, 157.

2. S. Turker, E. Onur, Y. Ozer. Nasal route and drug delivery systems. Pharmacy world and Science, 2004, 26, 137-142.

3. Beht et al. Optimization of systemic nasal drug delivery with pharmaceutical excipients, Adv Drug Del Rev, 1998, 29, 117-133.

4. Behl CR, Pimplaskar HK, Sileno AP, Meireles J, Romeoet VD. Effects of physicochemical properties and other factors on systemic nasal drug delivery. Adv Drug Deliv Rev 1998, 29,89-116.

5. Luessen HL, Verhoef JC, Boer AG, Junginger HE, De Leeuw BJ, Borchard G, Lehr CM. Multifunctional polymers for the peroral delivery of peptide drugs, in: E. Mathiowitz. D.E. Chickering III. C.M. Lehr (Eds.). Bioadhesive DrugDelivery Systems. Fundamentals. Novel Approaches and Development. Marcel Dekker. New York, 1999, 299- 339.

6. Dodane V, Amin KM, Merwin JR. Effect of chitosan on epithelial permeability and structure. Int J Pharm 1999, 182, 21-32.

7. Kotze AF, Luessen HL, Thanou M, Verhoef JC, De Boer AG, Junginger HE, Lehret CM.Chitosan and chitosan derivatives as absorption enhancers for peptide drugs across mucosal epithelia, in: E. Mathiowitz. D.E. Chickering III. C.-M. Lehr (Eds.), Bioadhesive Drug Delivery Systems. Fundamentals Novel Approaches, and Development. Marcel Dekker. New York. 1999, 341-386.

8. Bernkop-Schnurch A, Kast CE, Richter MF. Improvement in the mucoadhesive properties of alginate by the covalent attachment of cysteine. J Control Release 2001, 71, 277-285.

9. Wang J, Sakai S, Deguchi Y, Bi D, Tabata Y, Morimoto K. Aminated gelatin as a nasal absorption enhancer for peptide drugs: evaluation of absorption enhancing effect and nasal mucosa perturbation in rats. $\mathbf{J}$ Pharm Pharmacol 2002, 54,181- 188.

10. Nakamura K, Maitani Y, Lowman AM, Takayama K, Peppas NA, Nagai T. Uptake and release of budesonide from mucoadhesive, $\mathrm{pH}$ - studies have yielded useful information regarding nasal protein delivery with mucoadhesives. Garcia et al. ${ }^{44}$ showed that incorporation of cyanocobalamin into microcrystalline cellulose, dextran microspheres, and crospovidone resulted in significant improvement in bioavailability of cyanocobalamin relative to simple nasal solutions in rabbits. In another study, Hamman et al. ${ }^{45}$ examined the effect of the degree of quaternization of mucoadhesive N-trimethyl chitosan chloride (TMC) on nasal absorption enhancement of [14C]- mannitol in rats.

\subsection{Small organic molecules:}

Due to the rapid therapeutic action that can be achieved, medications used in emergency medical situations make ideal candidates for nasal drug delivery. One such drug, apomorphine is the drug of choice for treatment of on/offsyndrome in patients suffering from Parkinson's disease. Aqueous solution of the compound is reasonably well absorbed following nasal administration with a relative bioavailability of $45 \% .{ }^{46}$ Apart from increasing the mucosal contact time for the drug, mucoadhesive powder formulations had the added advantage of limiting oxidation due to aqueous environment. Although the nasal mucoadhesive formulations prepared with Carbopol 974P had better pharmacokinetic profiles than nasal solutions, the study showed that physicochemical characteristics of a drug molecule and its release from the polymer matrix could affect the rate and extent of drug absorption. Therefore, incorporation of a rapid release excipient (e.g., lactose) may be of relevance as demonstrated for apomorphine formulated with Carbopol 974P. ${ }^{47}$ Apomorphine absorption was fastest following intranasal administration of immediate release forms prepared with lactose compared to subcutaneous injections.

sensitive copolymers and their application to nasal delivery. J Control Release 1999, 61,329-335.

11. Park JS,. Oh YK, Yoon H, Kim JM, Kim CK. In situ gelling and mucoadhesive polymer vehicles for controlled intranasal delivery of plasmid DNA. J Biomed Mater Res 2002, 59,144-151.

12. Lansely AB. Mucociliary clearance and drug delivery via the respiratory tract. Adv Drug Deliv Rev 1993, 11, 299-327.

13. Tos M. Distribution of mucus producing elements in the respiratory tract. Difference between upper and lower airways. Eur J Respir Dis 1983, 64, 269-279.

14. Ridley D, Washington N, Wilson CG. Drug delivery to the buccal and nasal cavities, anatomical and physiological considerations, in: D. Duchêne (Ed.), Buccal and Nasal Administration as an Alternative Parenteral Administration. Editions de Sante. Paris. 1992, 29-39.

15. Stanley P, Wilson R, Greenstone M, McWilliam L, Coleet P. Effect of cigarette smoking on nasal mucociliary clearance and ciliary beat frequency. Thorax 1986, 41, 519-523.

16. Neutra MR, Forstner JF. Physiology of the Gastrointestinal Tract, Raven. New York. 1987, 975- 1009.

17. Thorton, DJ, Rousseau K, Muc Guckin MA. Structure and function of the polymeric mucins in airways mucus. Annual Review of Physiology, 2008, 44, 459-486.

18. Stefan G. Gastronaut: Adventures in Food for the Romantic, the Foolhardy, and the Brave, 2006, 68-69.

19. Strous GJ, Dekker J. Mucin-type glycoproteins. Crit Rev Biochem Mol Biol 1992, 27, 57- 92.

20. Pigman W, Moshera JL. The nature and function of the epithelial mucous glycoproteins, The Biology of the Cervix, University of Chicago Press. Chicago. 1973, 143-172.

21. Flynn GL, Yalkowsky SH, Roseman TJ. Mass transport phenomena and models: theoretical concepts. J Pharm Sci 1974, 63, 479-510. 
22. Sanders NN, DeSmedt SC, Demeester J. The physical properties of biogels and their permeability for macromolecular drugs and colloidal drug carriers. J Pharm Sci 2000 , 89, 835-849.

23. Jimenez-Castellanos MR, Zia H, Rhodes CT. Mucoadhesive drug delivery systems. Drug Dev Ind Pharm 1993, 19, 143- 194.

24. Duchene D, Touchard F, Peppas NA. Pharmaceutical and medical aspects of bioadhesive systems for drug administration. Drug Dev Ind Pharm, 1988, 14, 283-318.

25. Chang HS, Park H, Kelly P, Robinson JR. Bioadhesive polymers as platforms for oral controlled drug delivery: II. Synthesis and evaluation of some swelling, water-insoluble bioadhesive polymers. J Pharm Sci, 1985,74, 399- 405.

26. Longer MA, Robinson JR. Fundamental aspects of bioadhesion. Pharm Int 1986, 5,114- 117.

27. Derjaguin BV et al. On the relationship between the electrostatic and the molecular components of the adhesion of elastic particles to a solid surface. J Colloid Interface Sci, 1977, 58, 528-533.

28. Tobyn MJ, Johnson JR, Dettmar PW, Factors affecting in vitro gastric mucoadhesion: II. Properties of polymer. Eur J Pharm Biopharm, 1996, 42, 56-61.

29. Blanco-Fuente H, Anguiano S, Otero-Espinar FJ, Balnco-Mendez J. In-vitro bioadhesion of carbopol hydrogels. Int J Pharm, 1996, 142, $169-174$.

30. Park H, Robinson JR. Mechanism of mucoadhesion of poly(acrylic acid) hydrogels. Pharm Res, 1987, 4, 457-464.

31. Ponchel G, Touchard F, Duchene D, Peppas NA. Bioadhesive analysis of controlled-release systems: I. Fracture and interpenetration analysis in poly(acrylic acid-containing systems. J Control Release, 1987, 5, 129- 141.

32. Ponchel G, Touchard F, Duchene D, Peppas NA. Bioadhesive analysis of controlled-release systems: III. Bioadhesive and release behavior of metronidazole-containing poly(acrylic acid)hydroxypropyl methylcellulose systems. Int J Pharm, 1987, 38, 6570.

33. Hamman JH, Stander M, Kotze AF. Effect of the degree of quaternisation of $\mathrm{N}$-trimethyl chitosan chloride on absorption enhancement: in-vivo evaluation in rat nasal epithelia. Int J Pharm, 2002, 232, 235- 242.

34. Merkus FWHM, Schipper NGM, Hermens WAJJ, Romeijn SC, Verhoef JC. Absorption enhancers in nasal drug delivery: efficacy and safety. J Cont Rel, 1993, 24, 201-208.

35. Thananand M, Kumar DS, Shirwaikar A, Kumar R, Sampath Kumar D, Prasad RS. Preparation of mucoadhesive microspheres for nasal delivery by spray drying. Indian J Pharmaceutical Sciences, 2007, 69, 651-657.

36. Tiyaboonchai W. Chitosan Nanoparticles: A Promising System for Drug Delivery. Naresuan University Journal, 2003, 11, 51-66.

37. Lim ST, Martin GP, Berry DJ, Brown MB. Preparation and evaluation of the in-vitro drug release properties and mucoadhesion of novel microspheres of hyaluronic acid and chitosan. J Control Release, 2000, 66, 281-292.

38. Lim ST, Forbes B, Martin GP, Brown MB. In-vivo and in-vitro characterization of novel microparticulates based on hyaluronan and chitosan hydroglutamate. AAPS PharmSci- Tech, 2001, 2, article 20.

39. Cerchiara T, Luppi B, Bigucci F, Zecchi V. Chitosan salts as nasal sustained delivery systems for peptidic drugs. J Pharm Pharmacol, 2003, 55, 1623- 1627.

40. Kern W, Peters A, Fruehwald-Schultes B, Deininger E, Born J, Fehm HL. Improving influence of insulin on cognitive functions in humans. Neuroendocrinology, 2001, 74, 270-280.
41. Lemoine D, Deschuyteneer M, Hogge F, Preat V. Intranasal immunization against influenza virus using polymeric nanoparticles. J Biomater Sci. Polym Ed, 1999, 10, 805-825.

42. Westerink MAJ, Smithson SL, Srivastava N, Blonder J, Coeshott C, Rosenthal GJ, ProJuvantk (Pluronic F127R/ chitosan) enhances the immune response to intranasally administered tetanus toxoid. Vaccine, 2002, 20,711- 723.

43. Witschi C, Mrsny RJ. In-vitro evaluation of microparticles and polymer gels for use as nasal platforms for protein delivery. Pharm Res, 1999, 16, 382-390.

44. Ishikawa F, Murano M, Hiraishi M, Yamaguchi T, Tamai I, Tsuji A. Insoluble powder formulation as an effective nasal drug delivery system, Pharm Res, 2002, 19, 1097- 1104.

45. Garcia-Arieta A, Torrado-Santiago S, Goya L, Torrado JJ. Spraydried powders as nasal absorption enhancers of cyanocobalamin. Biol Pharm Bull, 2001, 24, 1411-1416.

46. Sam E, Jeanjean AP, Maloteaux JM, Verbeke N. Apomorphine pharmacokinetics in parkinsonism after intranasal and subcutaneous application. Eur J Drug Metab Pharmacokinet, 1995, 20, 27-33.

47. Ugwoke MI, Sam E, Van Den MG, Verbeke N, Kinget R. Bioavailability of apomorphine following intranasal administration of mucoadhesive drug delivery systems in rabbits. Eur J Pharm Sci, 1999, 9, 213-219.

48. Sharon N. Polysaccharides. Annu Rev Biochem, 1966, 35, 485-520.

49. Norris DA, Sinko PJ. Effect of size, surface charge, and hydrophobicity on the translocation of polystyrene microspheres through gastrointestinal mucin. J Appl Polym Sci, 1997, 63, 14811492 .

50. Martin L et al. The release of model macromolecules may be controlled by the hydrophobicity of palmitoyl glycol chitosan hydrogels. J Control Release, 2002, 80, 87-100.

51. Robinson JR, Longer MA, Veillard M. Bioadhesive polymers for controlled drug delivery. Ann N Y Acad Sci, 1987, 507, 307-314.

52. Walker RI. New strategies for using mucosal vaccination to achieve more effective immunisation. Vaccine, 1994, 12, 387-400.

53. FitzGerald D, Mrsny RJ. New approaches to antigen delivery .Crit Rev Ther Drug Carr Syst, 2000, 17, 1405- 1412.

54. Cano F, Plotnicky-Guilquin H, Nguyen TN, Liljeqvist S, Samuelson $\mathrm{P}$, Bonnefoy JY, Stahl S, Robert A. Partial protection to respiratory syncytial virus (RSV) elicited in mice by intranasal immunisation using live staphylococci with surface-displayed RSV-peptides. Vaccine, 2000, 18, 2743-2752.

55. Matsua K, Yoshikawa T, Asanuma H, Iwasaki T, Hagiwara Y, Chen $\mathrm{Z}$, Kadowaki S, Tsujimoto $\mathrm{H}$, Kurata $\mathrm{T}$, Tamura S. Induction of innate immunity by nasal influenza vaccine administered in combination with an adjuvant (cholera toxin). Vaccine, 2000, 18, 2713-2722.

56. Belshe RB, Mendelmn PM, Treanor J, Gruber WC, Piedra P, Bernstein DI, Hayden FG, Kotloff K, Zangwill K, Wolff M. The efficacy of live attenuated, cold adapted, trivalent, intranasal influenza virus vaccine in children. New Engl J Med, 1998, 338, 1405-1412.

57. Fehm HL, Smolnik R, Kern W, McGregor GP, Bickel U, Born J. The elanocortin melanocyte-stimulating hormone/ adrenocorticotropin 4 decreases body fat in humans. J Clin Endocrinol Metab, 2001, 86, $1144-1148$.

58. Vila A, Sanchez A, Tobyo M, Calvo P, Alonso MJ. Design of biodegradable particles for protein delivery. J Control Release, 2002, 78 ,
24. 